\title{
Apolipoprotein L2 contains a BH3-like domain but it does not behave as a BH3-only protein
}

\author{
J Galindo-Moreno', R lurlaro ${ }^{1}$, N El Mjiyad', J Díez-Pérez ${ }^{2,3}$, T Gabaldón $^{2,3,4}$ and C Muñoz-Pinedo ${ }^{*, 1}$
}

Apolipoproteins of the $\mathrm{L}$ family are lipid-binding proteins whose function is largely unknown. Apolipoprotein $\mathrm{L} 1$ and apolipoprotein L6 have been recently described as novel pro-death BH3-only proteins that are also capable of regulating autophagy. In an in-silico screening to discover novel putative BH3-only proteins, we identified yet another member of the apolipoprotein L family, apolipoprotein L2 (ApoL2), as a BH3 motif-containing protein. ApoL2 has been suggested to behave as a BH3-only protein and mediate cell death induced by interferon-gamma or viral infection. As previously described, we observed that ApoL2 protein was induced by interferon-gamma. However, knocking down its expression in HeLa cells did not regulate cell death induced by interferon-gamma. Overexpression of ApoL2 did not induce cell death on its own. ApoL2 did not sensitize or protect cells from overexpression of the BH3-only proteins Bmf or Noxa. Furthermore, siRNA against ApoL2 did not alter sensitivity to a variety of death stimuli. We could, however, detect a weak interaction between ApoL2 and Bcl-2 by immunoprecipitation of the former, suggesting a role of ApoL2 in a Bcl-2-regulated process like autophagy. However, in contrast to what has been described about its homologs ApoL1 and ApoL6, ApoL2 did not regulate autophagy. Thus, the role, if any, of ApoL2 in cell death remains to be clarified.

Cell Death and Disease (2014) 5, e1275; doi:10.1038/cddis.2014.237; published online 5 June 2014

Subject Category: Immunity

Bcl-2 family proteins regulate mitochondrial permeability to control apoptosis. These proteins induce or inhibit cell death, and they are associated with a growing number of pathologies, including cancer and immune diseases. ${ }^{1,2}$ For this reason, the search of new members of this family is of crucial importance. A subfamily of Bcl-2 homologs termed 'BH3-only proteins' comprises a growing number of proteins that only share a small motif of 15-21 amino acid residues. ${ }^{3,4}$ This region, known as the 'BH3-domain', is essential for the apoptotic function of Bcl-2 family proteins. The homology in this region is relatively loose, and only a few residues are conserved among the members of the family. For this reason, $\mathrm{BH} 3-$ only proteins have been identified by functional means rather than by sequence homology.

To identify novel BH3-only proteins we used a bioinformatics approach known as profile-based homology search. In brief, we constructed a so-called Hidden Markov Model (HMM) of the BH3-domain from the alignment of a set of proteins known to bear this domain. This HMM describes the probabilities of finding a given amino acid at a given position of the domain. This probabilistic model is then used to search in a sequence database for proteins that are likely to encode the same domain.

One of the proteins identified by this method was the apolipoprotein L2 (ApoL2). Two other members of this family, ApoL1 and ApoL6 have been described to behave as proapoptotic BH3-only proteins. ${ }^{5-7}$ Although the functions of these proteins are still unclear, proteins of this family have been shown to bind lipids and they have been suggested to work as pore-forming proteins in intracellular membranes, based on the ability of ApoL1 to form pores in the lysosomal membrane of trypanosomes. ${ }^{8,9}$ ApoL2 is highly homologous to ApoL1, and its BH3-like domain is very similar to those of ApoL1 and ApoL6. For these reasons, we explored the function of ApoL2 as a putative new BH3-only protein.

\section{Results}

Identification of novel BH3-containing proteins by using a profile-based homology search. Profile-based searches with profiles of $\mathrm{BH} 3$ domains as defined in ProSite ${ }^{10}$ and PFAM, ${ }^{11}$ as well as regular expression searches with motifs defined in the literature failed to provide satisfactory results in terms of specificity and sensitivity of detecting known human $\mathrm{BH} 3$ proteins (Table 1). Therefore, to efficiently identify novel putative BH3-only proteins, we collected the sequences of all human and mouse $\mathrm{BH} 3$ motifs annotated in Uniprot as well as those described in the literature, and aligned them to subsequently build an HMM for the BH3-domain (see Materials and Methods) (Figure 1a). This HMM provided better results in finding known $\mathrm{BH} 3$ than existing profiles at Pfam (Table 1), and was therefore used to search for

\footnotetext{
${ }^{1}$ Cell Death Regulation Group, IDIBELL (Institut d'Investigació Biomèdica de Bellvitge), Gran Via de L'Hospitalet 199, L'Hospitalet, 08908 Barcelona, Spain; ${ }^{2}$ Comparative Genomics Group, Centre for Genomics Regulation, Dr. Aiguader, 88, 08003 Barcelona, Spain; ${ }^{3}$ Universitat Pompeu Fabra (UPF), 08003 Barcelona, Spain and ${ }^{4}$ Institució Catalana de Recerca i Estudis Avançats (ICREA), Pg. Lluís Companys 23, 08010 Barcelona, Spain

*Corresponding author: C Muñoz-Pinedo, IDIBELL - Hospital Duran i Reynals 3a planta, Gran Via de L'Hospitalet 199, L'Hospitalet, 08908 Barcelona, Spain. Tel: +34 93260 7130; Fax: +34 93260 7426; E-mail: cmunoz@idibell.cat

Keywords: apolipoproteins L; Bcl-2 family proteins; BH3-only; interferon-gamma; autophagy

Abbreviations: ApoL, apolipoprotein L; FBS, fetal bovine serum; IFN- $\gamma$, interferon-gamma; TNF, tumor necrosis factor

Received 22.1.14; revised 14.4.14; accepted 22.4.14; Edited by G Melino
} 
putative novel BH3-containing proteins in the human proteome and genome.

Our screening identified BFK, a known Bcl-2 homolog originally left out from the list of proteins used to build the model, ${ }^{12}$ and $\mathrm{PXT1}$, a protein that has been recently described as a BH3-only protein that kills HeLa cells in a manner dependent of its $\mathrm{BH} 3$ motif $^{13}$ (Table 2). Another protein identified by this screening was the apolipoprotein $\mathrm{L} 2$ protein (Figure 1b, Table 2). Two apolipoproteins of the L family, ApoL1 and ApoL6, have been previously identified as BH3-only proteins. ${ }^{5-7}$ ApoL2, due to its homology with ApoL1 and ApoL6 has indeed been proposed to be a BH3-only protein. ${ }^{14}$ ApoL2 mRNA is ubiquitously expressed, according

Table 1 Summary of the results obtained from direct motif searches in the human proteome when using different strategies

\begin{tabular}{lccc}
\hline Motif search & $\begin{array}{c}\text { Hits in human } \\
\text { proteome }\end{array}$ & $\begin{array}{c}\text { Known } \\
\text { BCL's (TP) }\end{array}$ & $\begin{array}{c}\text { Sensitivity } \\
\text { (TP/TP + FN) }\end{array}$ \\
\hline 1. Youle et al. & 5908 & 13 & $68.4 \%$ \\
2. Liu et al. & 152 & 16 & $84.2 \%$ \\
3. Prosite & 28 & 9 & $47.36 \%$ \\
4. Novel HMM & 26 & 19 & $100 \%$ \\
\hline
\end{tabular}

First column indicates the search strategy: using a direct search with (1) The consensus BH3 motif (LXXXGD) as defined in Youle et al. ${ }^{4}(2)$ The extended motif (LXXX[GAS][DE]) used by Liu et al. ${ }^{7}$ in their identification of Apol6; (3) The motif defined by Prosite ${ }^{10}$ as of December 2008; and (4) an HMM-based search with the profile derived in this work. The following columns indicate, respectively, the number of total hits in the human proteome (Ensembl42 version), the number of known Bcl-2 family members identified of a total of 19 human members described in Youle et al. ${ }^{4}$ and Uniprot (2008), and, finally the sensitivity of the search as computed by dividing the total number of correctly identified Bcl-2 family members (TP) by the total number of known Bcl-2 family members $(T P+F N=19)$. TP and FN stand for True Positives and False Negatives, respectively to the database IST Online (Supplementary Figure 1). We checked that this protein is expressed in a variety of cell lines of different origins (Figure 2a), and highly expressed in HeLa cervical cancer cell line, as predicted due to its high expression in cervical cancer. ${ }^{15}$ ApoL2 is localized in HeLa cells outside the nucleus in a punctate state (Figure $2 b$ ) and it is not secreted (Figure 2c). Although it had been predicted to interact with membranes, ${ }^{16}$ we observed that it did not colocalize with mitochondrial, endoplasmic reticulum or lysosomal markers (Figure 2b).

ApoL2 is transcriptionally induced by interferon-gamma in a number of non-transformed tissues. ${ }^{14}$ In human bronchial epithelial cells its downregulation sensitized cells to cell death induced by IFN- $\gamma$, indicating that ApoL2 is an antiapoptotic protein in this context. ${ }^{14}$ We observed that in HeLa cells IFN- $\gamma$ induces ApoL2 (Figure 2d). However, when we downregulated ApoL2 using two different silencing sequences, we could not observe sensitization to cell death (Figure 2e).

ApoL2 is not a proapoptotic BH3-only protein. To check whether ApoL2 behaves as a proapoptotic Bcl-2 family member, we overexpressed ApoL2 in HeLa cells. Overexpression was confirmed by immunofluorescence (Supplementary Figure 2) and western blot (Supplementary Figure 3). We used Noxa and Bmf as proapoptotic BH3-only proteins, and verified that these proteins killed HeLa cells (Figure 3a). However, ApoL2 did not. We observed a trend of lower background death in cells overexpressing ApoL2, suggesting that ApoL2 is an antiapoptotic Bcl-2 family protein. To test this we overexpressed ApoL2 in combination with Noxa or Bmf. Our results indicate that ApoL2 confers a minor protection from Noxa (Figure 3a). However, this did not

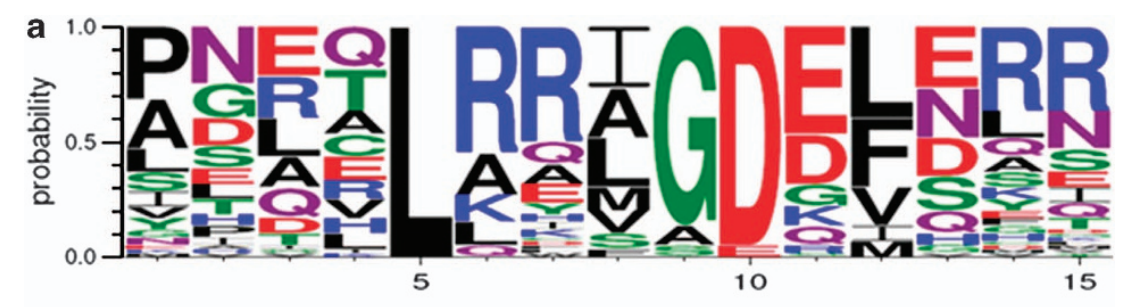

\begin{tabular}{|l|l|l|} 
& UniProt ID & BH3 motive sequence \\
\hline Bim & 043521 & IAQELRRIGDEFNAY \\
\hline Bad & Q92934 & YGRELRRMSDEFVDS \\
\hline Puma & Q9BXH1 & IGAQLRRMADDLNAQ \\
\hline Noxa & Q13794 & CATQLRRFGDKLNFR \\
\hline Bcl-Rambo & Q9BXK5 & MEDCLAHLGEKVSQE \\
\hline Beclin-1 & Q14457 & LSRRLKVTGDLFDIM \\
\hline ApoL6 & Q9BWW8 & NIDKLRALADDIDKT \\
\hline ApoL1 & O14791 & NIRRLRALADGVQKV \\
\hline ApoL2 & Q9BQE5 & HIRKLRALAEEVEQV \\
\hline
\end{tabular}

Figure 1 ApoL2 contains a BH3-like motif. (a) Logo representation of the protein profile used in the search for new BH3-domain proteins. The logo indicates the probability of finding a given amino acid at each of the 15 positions of the BH3-domain. Amino acids are represented by the one-letter code, and their height is proportional to the probability of appearing at a given position in the BH3-domain. (b) Alignment of ApoL2 BH3 motif with other $\mathrm{BH} 3$ motifs 
Table 2 Summary of the putative BH3-only proteins predicted using HMM

\begin{tabular}{|c|c|c|c|c|}
\hline Name & $\begin{array}{l}\text { UniProt } \\
\text { ID }\end{array}$ & NM ID & $\begin{array}{l}\text { Amino acid } \\
\text { number }\end{array}$ & Ensembl ID \\
\hline $\begin{array}{l}\text { Apolipoprotein L2 (ApoL2) } \\
\text { Arf-GAP with coiled-coil, ANK repeat and PH } \\
\text { domain-containing protein } 3 \text { (ACAP3) }\end{array}$ & $\begin{array}{l}\text { Q9BQE5 } \\
\text { Q96P50 }\end{array}$ & $\begin{array}{c}\text { NM_030882.2 and NM_145637.1 } \\
\text { NM_030649.2 }\end{array}$ & $\begin{array}{l}337 \\
834\end{array}$ & $\begin{array}{l}\text { ENSG00000128335 } \\
\text { ENSG00000131584 }\end{array}$ \\
\hline GDP-fucose protein O-fucosyltransferase 2 (POFUT2) & Q9Y2G5 & NM_015227.4 & 429 & ENSG00000186866 \\
\hline Phenylalanine-4-hydroxylase(PHA) & P00439 & NM_000277.1 & 452 & ENSG00000171759 \\
\hline Peroxisomal testis-specific protein 1 (PXT1) & J3KR74 & NM_152990.3 & 134 & ENSG00000179165 \\
\hline Uncharacterized protein C19orf55 (C19orf55) & Q2NL68 & NM_001039887 & 480 (putative) & ENSG00000167595 \\
\hline
\end{tabular}

The table shows the different identification codes of the gene from the major databases. Uniprot ID (http://www.uniprot.org/), NM ID (http://www.ncbi.nlm.nih.gov/) and Ensembl ID (http://www.ensembl.org/) as well as the number of amino acids of the protein
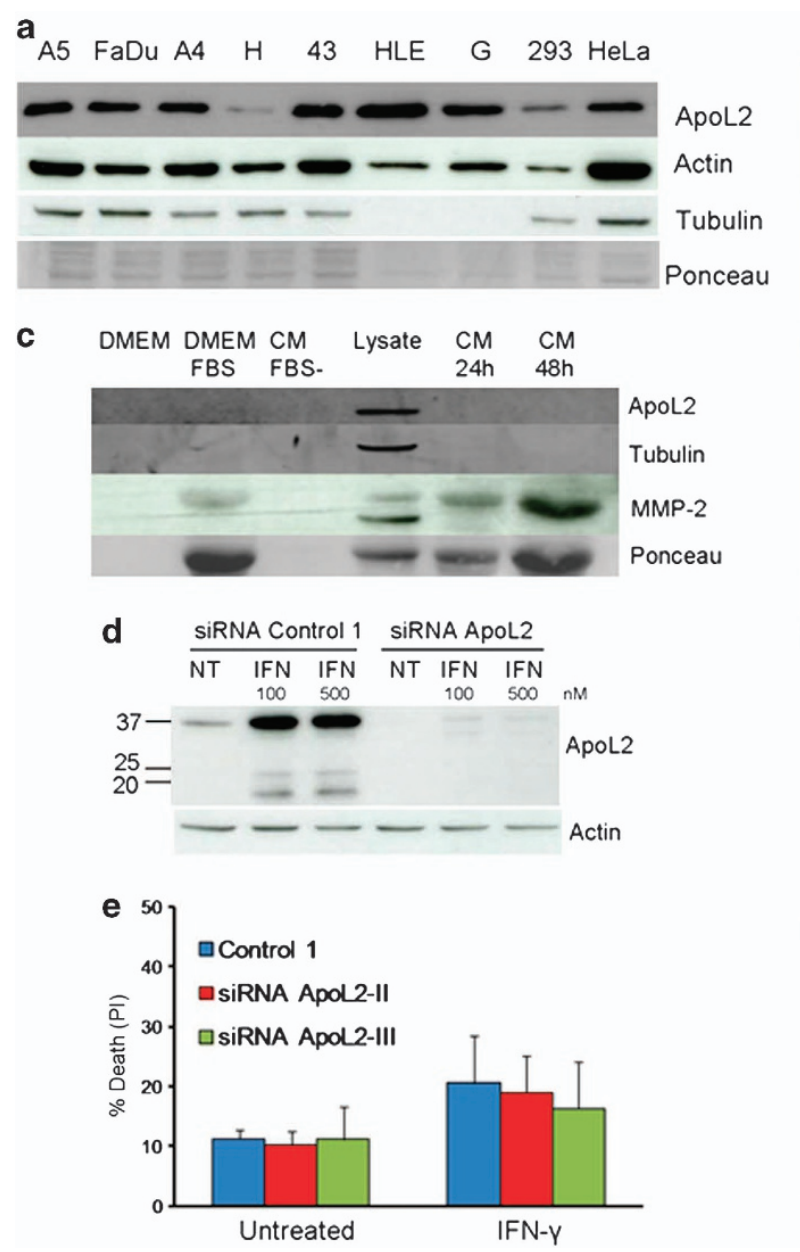

b
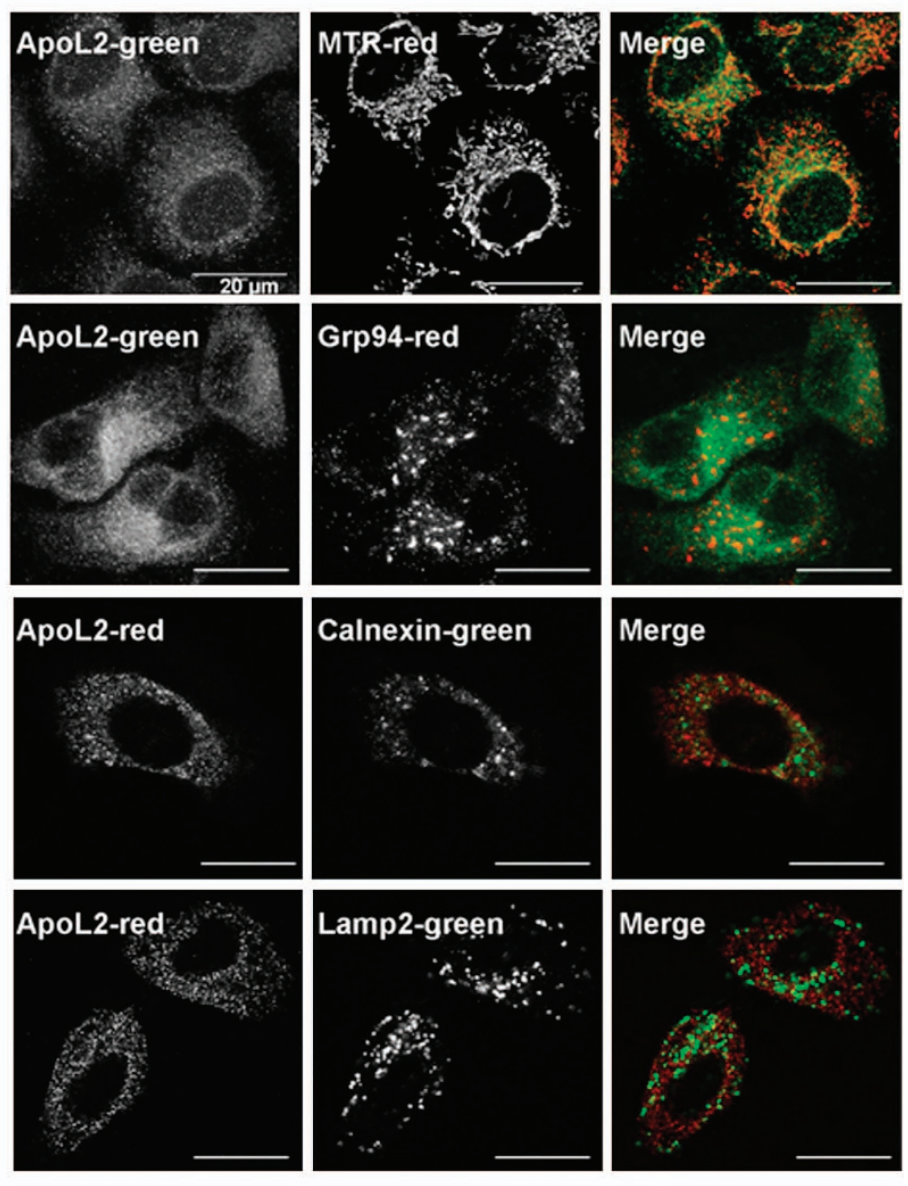

Figure 2 Cytosolic ApoL2 is widely expressed in different cell lines and induced by interferon-gamma. (a) ApoL2 expression was tested in different cell lines by western blot: A549 (A5), FaDu, A-431 (A4), HCT116 (H), 435P (43), HLE, HepG2 (G), HEK293 (293) and HeLa cells. (b) Intracellular localization of ApoL2. HeLa cells were stained with MitoTracker red (MTR) as mitochondrial marker. Antibodies against Grp94 and Calnexin were used as endoplasmic reticulum markers. Lysosomal localization was studied using Lamp-2 antibody. Scale bars of $20 \mu \mathrm{m}$ are shown. (c) ApoL2 is not secreted. Western blot of trichloroacetic acid (TCA)-concentrated medium or cell lysate is shown. DMEM and DMEM complemented with FBS were used as controls. CM FBS - : conditioned medium of HeLa cells grown for $48 \mathrm{~h}$ in DMEM without FBS. Lysate: cell lysate of HeLa cells grown in FBS containing medium for $48 \mathrm{~h}$. CM: conditioned medium of HeLa cells grown in FBS containing medium for indicated times. Antibodies against ApoL2, tubulin, actin and the secreted protein metalloproteinase-2 (MMP-2) were used for immunoblotting. (d) HeLa cells were transfected with siRNA ApoL2-II, treated with interferon-gamma (IFN) at 100 or $500 \mathrm{nM}$ for $24 \mathrm{~h}$ and collected for western blot. NT means non treated. (e) HeLa cells were transfected with siRNA control 1 or siRNA against ApoL2 and treated with IFN- $\gamma 100 \mathrm{nM}$ for $72 \mathrm{~h}$. Cell death was measured by PI incorporation at the flow cytometer. Figure shows average and S.E.M. of three independent experiments

reach statistical significance $(n=3)$. Bcl-2 was employed as a control (expression checked in Supplementary Figure 3) and it protected from Noxa and Bmf.
Next we analyzed whether ApoL2 would regulate cell death induced by a variety of stimuli, either by behaving as an antiapoptotic protein as described ${ }^{14}$ or as a proapoptotic 
a

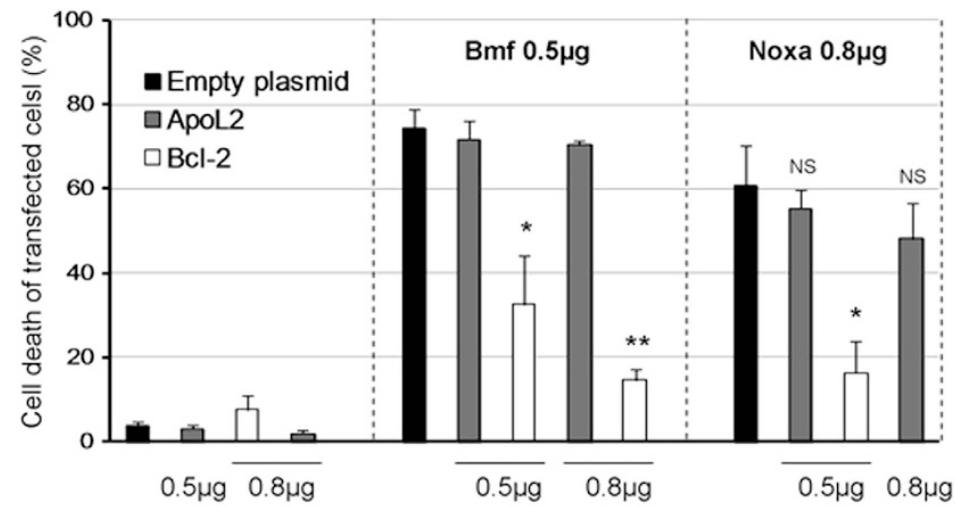

b

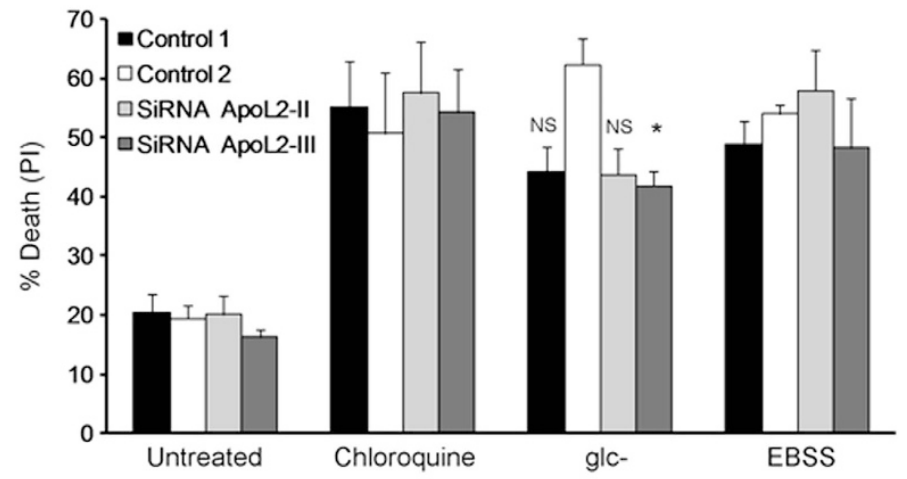

C

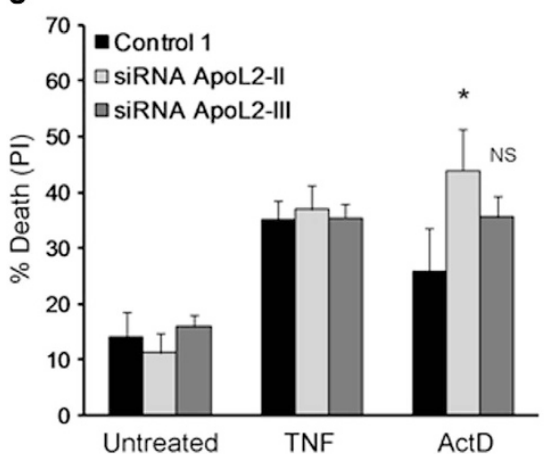

d

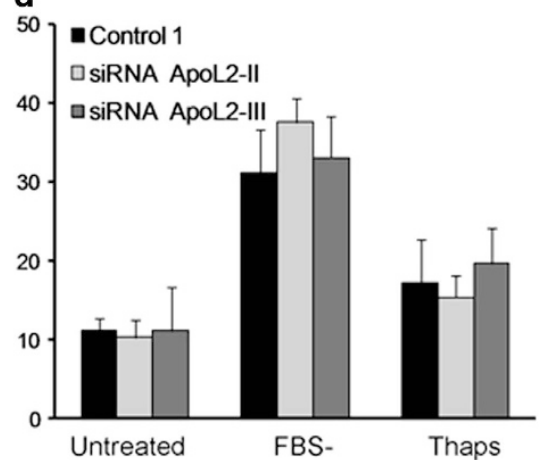

Figure 3 ApoL2 does not regulate cell death of HeLa cells. (a) Plasmids encoding two different BH3-only proteins ( $0.5 \mu \mathrm{g}$ of Bmf and $0.8 \mu \mathrm{g}$ of Noxa plasmids) were cotransfected with ApoL2 or Bcl-2 and analyzed by microscopy. GFP $(0.3 \mu \mathrm{g})$ was used as transfection marker. ApoL2 and Bcl-2 plasmids were used at amounts shown. Empty plasmid was used to normalize the amount of transfected DNA. Dead green cells were scored by shrunk morphology and counted from images using fluorescence microscopy. Figure shows average and S.E.M. of three experiments. For statistical analysis, each ApoL2 or Bcl-2 overexpressing condition has been compared with the empty plasmid condition transfected with the same BH3-only protein. NS, nonsignificant. (b, c, d). HeLa cells were transfected with different control siRNAs or siRNA against ApoL2 and then treated with chloroquine, deprived of glucose (glc - ), incubated in starvation buffer (EBSS) or treated with tumor necrosis factor (TNF) or actinomycin D (ActD) for $24 \mathrm{~h}(\mathbf{b}, \mathbf{c})$, or deprived of serum (FBS - ) or treated with thapsigargin (Thaps) for $72 \mathrm{~h}(\mathrm{~d})$. Cell death was measured by PI incorporation by flow cytometry. Figure shows average and S.E.M. of three (d) or five experiments (b, c). Asterisks or NS (nonsignificant) denote significance versus Control 2 (b) or Control 1 (c)

BH3-only protein like ApoL1 and ApoL6. We knocked down ApoL2 using different siRNA sequences and treated HeLa cells with the endoplasmic reticulum stressor thapsigargin, the DNA damaging agent actinomycin D, the lysosomal inhibitor chloroquine, or starvation of serum, glucose or serum/amino $\mathrm{acid} /$ vitamins (culture in EBSS buffer) (Figures $3 \mathrm{~b}-\mathrm{d}$ ). We only observed a minor difference in cell death induced by actinomycin $D$ that was significant when cells were depleted of ApoL2 using one siRNA oligo but not the second one. ApoL2 has been shown to be induced by TNF. ${ }^{6}$ We did not observe induction of ApoL2 upon TNF treatment in HeLa or
293T cells (Supplementary Figure 4). In addition, we treated HeLa cells with TNF in the presence of cycloheximide to induce cell death, and we did not observe any difference when ApoL2 was silenced (Figure 3c).

ApoL2 interacts weakly with $\mathrm{Bcl}-2$ but it does not regulate autophagy. We could not detect a role of ApoL2 in cell death. However, not all $\mathrm{BH}$-only proteins described to date regulate cell death. Some proteins like Beclin-1 regulate autophagy through its interaction with $\mathrm{Bcl}-2$ family proteins. ApoL6, which induces cell death and inhibits autophagy, has 
been shown to bind Bcl-xL. ${ }^{17}$ We thus tested whether endogenous ApoL2 interacted with other BH3-containing proteins. We immunoprecipitated ApoL2 and blotted for multidomain $\mathrm{Bcl}-2$ family proteins. Bcl-2 was reproducibly immunoprecipitated with ApoL2 (Figure 4a). We were unable to immunoprecipitate endogenous $\mathrm{Bcl}-2$ under the same conditions (not shown). For these reasons, to confirm these interactions in a different manner we overexpressed HA-tagged $\mathrm{Bcl}-2 .{ }^{18}$ Under these conditions, we were unable to immunoprecipitate ApoL2 with anti-HA antibody (Figure 4b) or to detect HA upon immunoprecipitation of ApoL2, neither in HeLa nor in 293T cells (not shown). We next checked whether the weak interaction between ApoL2 and Bcl-2 (detected only using endogenous proteins) would alter the sensitivity of HeLa cells to the Bcl-2 and $\mathrm{Bcl}-\mathrm{xL}$ inhibitor ABT-737. Downregulation of ApoL2 did not alter the amount of cell death induced by ABT-737 (Figure 5a).

One possibility is that ApoL2, by interacting with Bcl-2 or signaling lipids, would regulate cell proliferation. We tested this and could not observe any effects on cellular proliferation by downregulation of ApoL2 (Figure 5b). We next investigated whether ApoL2 could act like Beclin-1 or ApoL6 regulating autophagy. ${ }^{17}$ We downregulated ApoL2 (Figures 6a and b) and measured basal autophagy (lipidation of LC3 and degradation of $p 62$ in the presence or absence of protease inhibitors) and starvation-induced autophagy (same measurements after incubation in starvation buffer EBSS). Our results indicate that ApoL2 does not alter basal or starvation-induced autophagic flux as measured by levels of LC3-II (Figures 6a and $c$ ). We did observe a significant reduction of $p 62$ levels after ApoL2 was downregulated, suggesting that this protein regulates basal autophagy (Figures 6a and d), but this was not accompanied by a difference in levels of LC3-II at these conditions (Figure 6c).

Altogether, our data indicate that ApoL2 is not a classical BH3-only protein, and its exact function in cell death by interferon treatment remains to be determined.

\section{Discussion}

BH3-only proteins do not share a high degree of homology between them, and it is possible that the $\mathrm{BH} 3$-domain arose either randomly during evolution or by a process of convergent evolution. ${ }^{19}$ Moreover, the $\mathrm{BH} 3$-domain is not extremely well conserved even among $\mathrm{Bcl}-2$ family proteins that share more domains than the $\mathrm{BH} 3{ }^{3}{ }^{3}$ Many $\mathrm{BH} 3-o n l y$ proteins have not been identified by sequence, but on the basis of their interaction with $\mathrm{Bcl}-2$ family proteins. Other members of this family have been found to be proapoptotic proteins and the putative $\mathrm{BH} 3$-motif was identified later. We have performed here a search based on a newly-generated protein composition profile that was shown to identify all known $\mathrm{BH} 3-$ only proteins plus few additional candidates in the human genome.

Our screening identified the protein PXT1, which has a $\mathrm{BH} 3-$ like domain. This protein has been described to induce cytochrome $c$ release and apoptosis in HeLa cells in a manner dependent on its $\mathrm{BH} 3$ motif. ${ }^{13}$ In addition, apolipoprotein L2 (ApoL2) caught our attention due to the recent description of ApoL2 homologs as BH3-only proteins. ApoL1, the founding member of the family, was identified as a component of a class of high density lipoproteins (HDL) in human blood. ${ }^{20}$ In subsequent years, a number of homologous proteins have been described: the apolipoprotein $L$ family comprises six members in humans and $8-14$ members in rodents. ${ }^{16,21}$ ApoL1 is the only member of the family expected to be secreted, and when internalized by trypanosomes it generates pores in their lysosomal membrane. ${ }^{8}$ ApoL1 and ApoL6 also kill mammalian cells when overexpressed, and it has been proposed that all members of the family could share this ability with these two proteins. ${ }^{9}$ Induction of cell death by ApoL1 and ApoL6 was prevented when their $\mathrm{BH} 3$ motif was deleted. ${ }^{5,7}$ Both proteins bind lipids;, ${ }^{5,7}$ interestingly, ApoL1 binds cardiolipin which is a lipid required for permeabilization of liposomes by $\mathrm{Bcl}-2$ family members. ${ }^{22}$ ApoL6 binds $\mathrm{Bcl}-\mathrm{xL}$
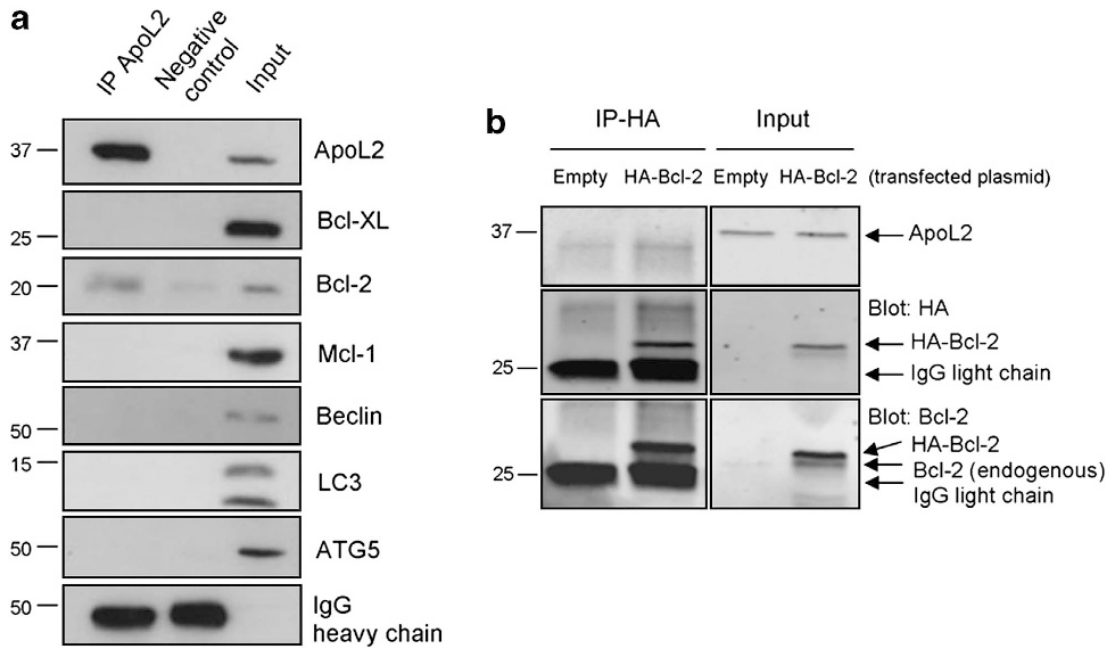

Figure 4 Immunoprecipitation of ApoL2 in HeLa cells. (a) Endogenous ApoL2 was immunoprecipitated (IP) and the presence of the indicated proteins was assayed by western blot. Blots from a single experiment representative of three independent experiments are shown. (b) HeLa cells were transfected with HA-Bcl-2 or empty vector. AntiHA was used for immunoprecipitation and the presence of ApoL2, Bcl-2 and HA was assayed by western blot. Panel shown is representative of three independent experiments. Left and right panels were cropped from the same films 
a

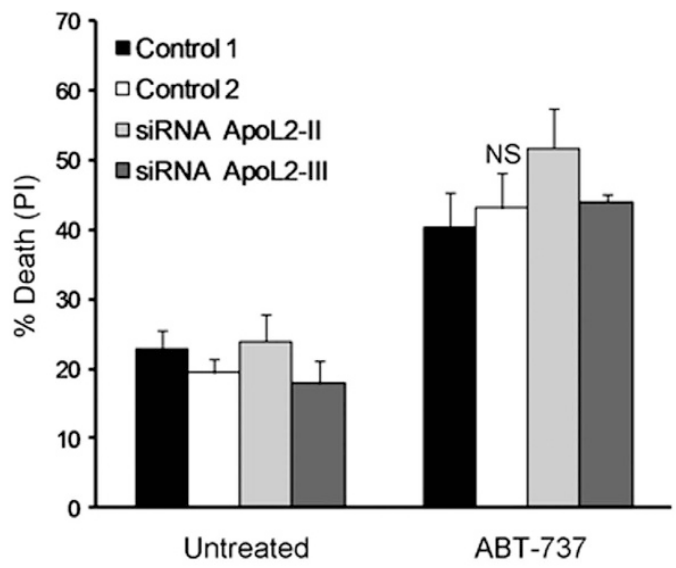

b

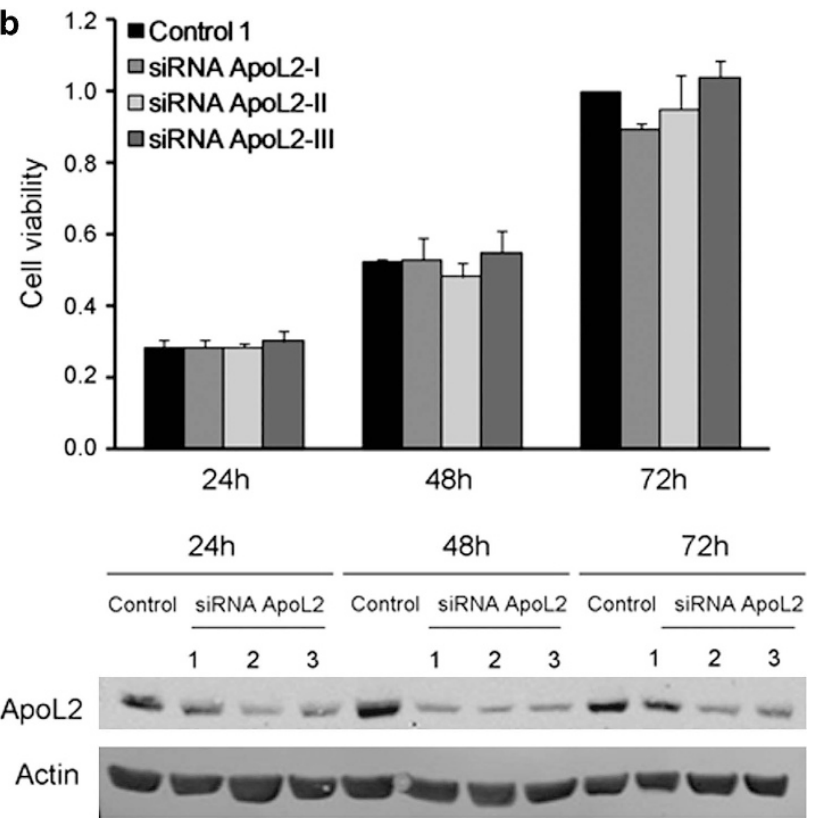

Figure 5 ApoL2 does not regulate cell proliferation or sensitivity to ABT-737. (a) HeLa cells were transfected with control SiRNA or siRNA targeting ApoL2 and then they were treated with the $\mathrm{BH} 3$ mimetic ABT-737 at $30 \mu \mathrm{M}$ for $24 \mathrm{~h}$. Cell death was measured by PI incorporation and flow cytometry. Panel shows average and S.E.M. of five independent experiments. (b) HeLa cells were transfected with control 1 siRNA or siRNAs against ApoL2 and growth analysis was performed at indicated time points by crystal violet coloration. The lower panel shows western blot analysis of ApoL2 silencing over time

and it regulates autophagy..$^{17}$ So indeed, many similarities exist between some members of the apolipoprotein $L$ family and 'classic' Bcl-2 family proteins.

ApoL2 has been shown to be antiapoptotic in primary cells treated with interferon-gamma. ${ }^{14}$ Recently, ApoL2 has been identified as a protein that translocates to mitochondria in cells infected with H3N2 swine influenza virus. ${ }^{23}$ These two facts, together with the description of other proteins of the family as BH3-only proteins has led to propose that ApoL2 has a role in apoptosis, which we have not been able to confirm. It is possible that the aspartic residue in position 10 of the motif (Figure 1) is essential for their proapoptotic function. The function of other $\mathrm{Bcl}-2$ family members with a glutamic acid in that position, Bcl-Rambo (Bcl2L13) and Bcl-G (Bcl2L14), is still not fully defined, but their main function may be unrelated to cell death. ${ }^{24,25}$

We have detected a weak interaction between $\mathrm{Bcl}-2$ and ApoL2. However, this did not alter apoptosis induced by many stimuli or starvation-induced autophagy. We did observe a basal regulation of p62, an autophagic protein, which could suggest that this protein regulates autophagy under certain conditions due to its interaction with $\mathrm{Bcl}-2$. On the other hand, $\mathrm{Bcl}-2$ regulates multiple metabolic pathways, $\mathrm{Ca}^{2+}$ stores in the endoplasmic reticulum, mitochondrial morphology and DNA repair. ${ }^{26,27}$ We have not explored here the possibility that ApoL2 regulates these functions of $\mathrm{Bcl}-2$. Nonetheless, it is also possible that the ApoL2 has a cell-type or stimulusdependent role on cell death that according to our data is not general or ubiquitous.

\section{Materials and Methods}

Building the HMM. Sequences of vertebrate proteins annotated with the $\mathrm{BH} 3-d o m a i n$ in Uniprot and the literature as of November 2008 (Bcl-2, Bcl-XL, Bcl-w, Mcl-1, Bcl-Rambo, Bcl-G, Bax, Bak, Bok, Bim, Bid, Bad, Bmf, Noxa, Hrk, Puma, Bik, Blk, Mule, Spike, Nix, BNIP3, Map-1, Cul7, Beclin-1, p53, ApoL6, ApoL1 and AVEN) were aligned with MAFFT. ${ }^{28}$ A 15-residue long region from the alignment containing the annotated $\mathrm{BH} 3$ domains was selected using trimAl $1.3{ }^{29}$ and a HMM model was built for the region using HMMER v1.8.5. ${ }^{30}$ HMMER was used to search in the entire human proteome, as retrieved from Ensembl database version $50 .{ }^{31}$ To detect the domain in putative unpredicted proteins we ran Exonerate $^{32}$ using that profile over the genome sequence. The results were compared with similar searches using the profiles available at PFAM (which rendered only already-annotated proteins) and Prosite databases, as well as with regular expression searches with motifs described in the literature (Table 1).

Cell culture and treatments. HeLa cells from American Type Culture Collection and 293T were cultured in pyruvate-free high glucose Dulbecco's Modified Eagle's Medium (DMEM; Gibco Life Technologies, Waltham, MA, USA) supplemented with $10 \%$ fetal bovine serum (FBS; Invitrogen, Carlsbad, CA, USA), $200 \mathrm{mg} / \mathrm{ml}$ of penicillin, $100 \mu \mathrm{g} / \mathrm{ml}$ of streptomycin and glutamine $2 \mathrm{mM}$ (hereafter referred to as PSQ). Cells were maintained at $37^{\circ} \mathrm{C}$ and in a $5 \% \mathrm{CO}_{2}$ atmosphere. Cell maintenance is based on three splits per week using trypsin EDTA-Solution $0.05 \%$ (Invitrogen). HeLa cells were plated at a concentration of $150000 / \mathrm{ml}$ in $6-$ or 12-well plates and treated $24 \mathrm{~h}$ later, when they reached the concentration of $500000 / \mathrm{ml}$. 293T cells were plated at a concentration of $1 \times 10^{6} / \mathrm{ml}$ in $10 \mathrm{~cm}$ plates for transfections.

Before glucose-deprivation or FBS-deprivation treatments, cells were washed twice with FBS-free, pyruvate-free DMEM medium without glucose (Gibco Life Technologies) or high-glucose, FBS-free DMEM, respectively. Glucose-deprivation treatment is performed in PSQ-containing, glucose-free DMEM medium without glucose supplemented with $10 \%$ dialyzed FBS. FBS-deprivation treatment was performed in PSQ-containing high-glucose DMEM. For induction of cell death by starvation in Earle's Balanced Salt Solution (EBSS, Gibco Life Technologies), cells were washed twice with EBSS before treating with EBSS supplemented with Hepes $25 \mathrm{mM}$.

ABT-737 (Selleck Chemicals, Houston, TX, USA) is used at $30 \mu \mathrm{M}$, chloroquine, thapsigargin and actinomycin D (Sigma-Aldrich, St. Louis, MO, USA) at $100 \mu \mathrm{M}$, $100 \mu \mathrm{g} / \mathrm{ml}$ and $50 \mathrm{nM}$, respectively, interferon-gamma (Novus Bionova, Madrid, Spain) at $100 \mathrm{ng} / \mathrm{ml}$. TNF- $\alpha$ (Peprotech, Le-Perray-en-Yvelines, France, $10 \mathrm{ng} / \mathrm{ml}$ ) is added in combination with $10 \mu \mathrm{M}$ cycloheximide (Sigma-Aldrich) to induce cell death.

For autophagy induction, cells were incubated in home-made EBSS (potassium chloride $400 \mathrm{mg} / \mathrm{ml}$, sodium bicarbonate $2.2 \mathrm{~g} / \mathrm{ml}$, sodium chloride $6.8 \mathrm{~g} / \mathrm{ml}$, NaH2PO4-H2O $140 \mathrm{mg} / \mathrm{ml}$, D-Glucose $1 \mathrm{~g} / \mathrm{ml}$ ) supplemented with $25 \mathrm{mM}$ Hepes. EBSS-Hepes treatment is performed after washing the cells twice with EBSS.

Autophagy flux was blocked by adding the protease inhibitors pepstatin $A$ and E64d (Sigma, St. Louis, MO, USA, $10 \mu \mathrm{M}$ each) simultaneously with the treatments.

Cell viability. For analysis of viability, cells were harvested by combining floating cells in the medium and adherent cells detached by trypsinization, and subjected to FACS analysis to detect incorporation of propidium iodide $1 \mu \mathrm{g} / \mathrm{ml}$ 
a
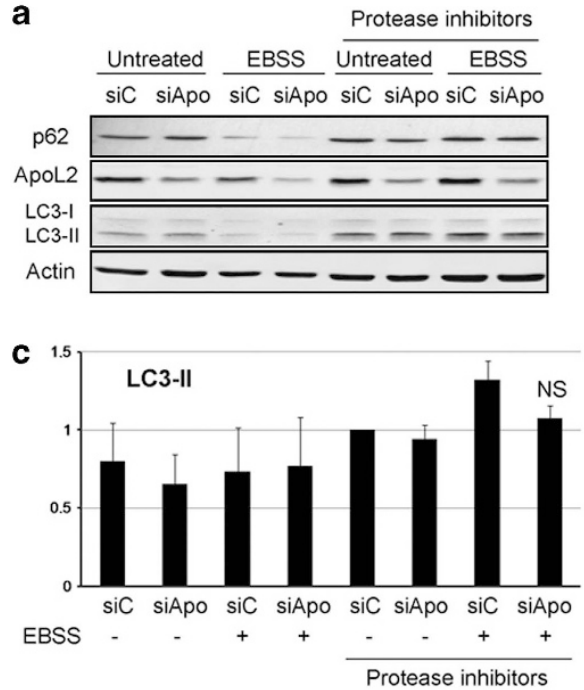

b

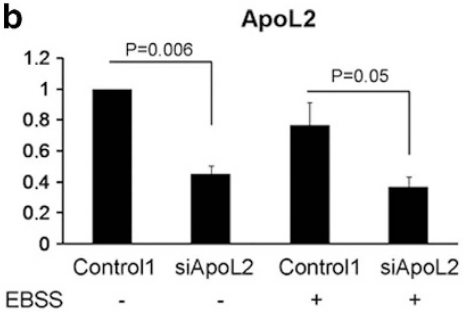

d

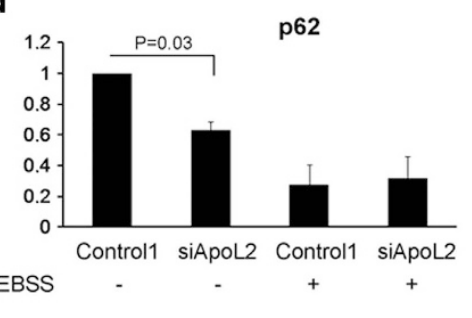

Figure 6 ApoL2 does not regulate autophagy. HeLa cells were transfected with siRNA Control 1 (labeled as siC) or siRNA against ApoL2-II (labeled as siApo) for 48 h and then the medium was changed or they were incubated with EBSS for $6 \mathrm{~h}$ to induce autophagy. The protease inhibitors pepstatin and E64D (10 $\mu \mathrm{M}$ each) were used to block autophagic flux. A representative western blot is shown in $\mathbf{a}$. ApoL2 levels were quantified and are shown in $\mathbf{b}$ : data were weighted to ponceau or actin and then normalized against Control 1-transfected untreated cells. (c) Quantification of relative LC3-II levels: data were weighted to ponceau or actin and then normalized against Control 1-transfected HeLa cells with protease inhibitors as control of basal autophagy. (d) Quantification of relative p62 levels: data were weighted to ponceau or actin and then normalized against Control 1-transfected untreated cells. Graphs show average and S.D. of three independent experiments

(10 min incubation in PBS) using Gallios Flow Cytometer Beckman Coulter. Data were analyzed using FlowJo software, version 7.6.4.

Cell viability and number was additionally measured by crystal violet coloration. After the indicated treatments, cells were covered with staining solution $(0.2 \%$ crystal violet, $2 \%$ EtOH solution) and incubated for 20 min at room temperature. Cells were rinsed twice with PBS and once with water and let dry for $16 \mathrm{~h}$. Crystal violet-stained cells were then resuspended in $10 \%$ SDS and absorbance was read at $595 \mathrm{~nm}$ in a BioTek (Winooski, VT, USA) PowerWave XS microplate spectrophotometer.

Western blotting. Cell pellets were resuspended in RIPA buffer (Thermo Scientific, Waltham, MA, USA) or lysis buffer (0.06 M Tris, $2 \%$ SDS) containing protease inhibition cocktail (Roche, Basel, Switzerland) and phosphatase inhibitors (PhosSTOP, Roche) and they were then sonicated.

For trichloroacetic acid (TCA) precipitation, the acid (Merck, Darmstadt, Germany) was added to the sample at a final concentration of $13 \%$, mixed thoroughly and incubated overnight at $4{ }^{\circ} \mathrm{C}$ under rotation. The mixture was centrifugated $\left(16000 \times \mathrm{g}, 15 \mathrm{~min}, 4^{\circ} \mathrm{C}\right)$, and the supernatant was discarded. The pellet was resuspended in RIPA buffer.

Protein quantification was performed using Pierce BCA protein assay kit, following the manufacturer's instructions. $40 \mu \mathrm{g}$ of protein were diluted in $10 \mu \mathrm{l}$ of laemmli buffer $4 \times(63 \mathrm{mM}$ Tris- $\mathrm{HCl}, 10 \%$ glycerol, $2 \%$ SDS, $0.01 \%$ bromophenol blue and $5 \%$ 2-mercaptoethanol), and PBS was added until $40 \mu \mathrm{l}$ of total volume. Lysates were boiled for $10 \mathrm{~min}$ at $96^{\circ} \mathrm{C}$ and loaded in a $12 \%$ acrylamide gel. Miniprotean (Bio-Rad, Hercules, CA, USA) electrophoresis tank was used to perform the electrophoresis assay. Proteins were transferred to polyvinylidene fluoride (PVDF, Millipore, Darmstadt, Germany) or nitrocellulose membranes (Bio-Rad) through semi-dry transfer ( $1 \mathrm{~h}$ at $0.2 \mathrm{~A} /$ membrane). Transfer validation and loading charge control was checked by ponceau dye (Sigma). PVDF membranes were blocked with $5 \%$ nonfat dry milk in Tween Tris-buffered saline (TTBS) and processed for immunoluminiscence. Nitrocellulose membranes were blocked with Odyssey Blocking Buffer (LI-COR Biosciences, Lincoln, NE, USA) and processed for immunofluorescence using Odyssey Fc Imaging system. Primary and secondary antibodies were incubated for $1 \mathrm{~h}$ at room temperature or overnight at $4{ }^{\circ} \mathrm{C}$, in $5 \%$ milk TTBS. Three 10-min TTBS washes in the shaker were performed before developing by enhanced chemiluminescence (ECL; Pierce, Waltham, MA, USA) or scanning the membrane using Odyssey Imaging System. Quantification of band intensity was performed with Fij//mage J software $1.47 \mathrm{~b}$.

Primary antibodies used for western blotting were: anti-actin (ICN clone 4), polyclonal anti-ApoL2 (Sigma, HPA001078), anti-tubulin (Sigma, Clone TUB 2.1). polyclonal antibody against p62 (Progen, Heidelberg, Germany), polyclonal
anti-LC3 (Abcam, Cambridge, UK), anti-HA (Sigma, clone HA-7), anti-Bcl-xL (Cell Signaling, Beverly, MA, USA, 54H6), anti-Bcl-2 (Santa Cruz, Dallas, TX, USA, 100), polyclonal anti-Mcl-1 (Santa Cruz, sc-819), polyclonal anti-Atg5 (CosmoBio, Tokyo, Japan), anti-beclin-1 (BD Biosciences, Franklin Lakes, NJ, USA, 20/Beclin). HRP secondary antibodies were: antimouse and anti-rabbit (Zymax, Bideford, UK) or anti-guinea pig (Abcam). IRDye secondary anti-bodies against mouse or rabbit (IRDye $800 \mathrm{CW}$ donkey anti-rabbit IgG1 $1 / 15.000$ or IRDye $680 \mathrm{LT}$ anti-mouse IgG $1 / 20000$ ) were from LI-COR Biosciences.

Plasmids and transient transfection. ApoL2 cDNA (NM_030882.2) was purchased from Origene and subcloned into ampicillin resistant pcDNA3.1 plasmid using EcoR1 and BamH1 restriction enzymes. Invitrogen PureLink kit was used to extract the plasmid from competent bacteria (Promega, Fitchburg, WI, USA).

For death experiments, HeLa cells were transfected in six-well plates, using $4 \mu \mathrm{l}$ of Genejuice (Novagen, Darmstadt, Germany) and $2 \mu \mathrm{g}$ of total DNA. To normalize until $2 \mu \mathrm{g}$, we completed with the empty plasmid pcDNA 3.1. pcDNA 3.1-Bcl-2 plasmid was generously provided by Dr. Jean-Ehrland Ricci (Nice, France). HANoxa and pcDNA 3.1-Bmf were provided by Professor Seamus Martin (Dublin, Ireland). pcDNA 3.1-HA-Bcl-2 and pcDNA 3.1-HA-Bcl-xL plasmids from Dr. Douglas Green's laboratory (Memphis, TN, USA) were used in the immunoprecipitation assay. Cell death was analyzed by counting GFP positive dead cells against total GFP positive cells using an Olympus IX70 inverted microscope. For immunoprecipitation, HeLa cells were transfected in $10 \mathrm{~cm}$ dishes, using $3 \mu \mathrm{g}$ of polyethylenimine linear (PEl; Polyscience Europe, Heidelberg, Germany) per $\mu \mathrm{g}$ of DNA. Cotransfection was performed using $10 \mu \mathrm{g}$ of ApoL2 and $10 \mu \mathrm{g}$ of HA-Bcl$\mathrm{xL}$ or HA-Bcl-2 plasmid.

siRNA transfection. Cells were transfected at a density of $300000 / \mathrm{ml}$ using $1.5 \mu \mathrm{l}$ of DharmaFECT 1 (Dharmacon, Lafayette, CO, USA) per milliliter of total volume and following manufacturer's instructions. SiRNA concentration was $100 \mathrm{nM}$. After $24 \mathrm{~h}$, medium was replaced with growth medium. Control sequences were: 5'-GUAAGACACGACUUAUCGC[dT][dT] ('control 1') and an ON-TARGET plus siRNA pool of 4 oligos against mouse RIPK (Dharmacon; 'control 2'). Three different siRNA sequences were used against ApoL2: ApoL2-I (GCGGCAC CAAUGUAGCAAA[dT][dT]), ApoL2-II (CAGUGUGGUAGAACUAGUA[dT][dT]) and ApoL2-III (CAAUGUUCUUACCUUAGUU[dT][dT]).

Immunofluorescence. Cells were cultured on glass coverslips pretreated with poly-L-Lysine (Sigma). After $24 \mathrm{~h}$ they were incubated for $15 \mathrm{~min}$ in culture medium at $37^{\circ} \mathrm{C}$ and $5 \% \mathrm{CO}_{2}$ with MitoTracker red $200 \mathrm{nM}$ (Invitrogen) before 
fixing, or they were directly fixed with a fresh $4 \%$ solution of paraformaldehyde for $20 \mathrm{~min}$. Cells were then incubated with blocking buffer: $0.05 \%$ Triton, $3 \%$ BSA in PBS for $1 \mathrm{~h}$ and kept overnight at $4{ }^{\circ} \mathrm{C}$ with primary antibodies diluted $1: 200$ in blocking buffer: Ab rabbit anti-ApoL2 (Sigma), mouse anti-Lamp-2 (BD pharmigen, Franklin Lakes, NJ, USA, CD107b, 555803), mouse anti-Calnexin (Santa Cruz, E-10, sc-46669), goat anti-GRP94 (Santa Cruz, C-19, sc-1794). Cells were incubated with secondary antibodies Alexa Fluor 568 red and 488 green (Life Technologies, Carlsbad, CA, USA) diluted 1:400 in blocking buffer for $1 \mathrm{~h}$. Then they were mounted in Vectashield solution (Vector laboratories, Burlingame, CA, USA) on microscope slides and visualized on a Leica TCS SP5 Spectral Confocal microscope with a HCX PL APO lambda blue $\times 631.4$ oil objective lens. Acquisition software was LEICA (Wetzlar, Germany) Application Suite Advanced Fluorescence (LAS AF) version 2.6.0.7266 and pictures were analyzed with Fij/lmage $\mathrm{J}$ software.

Immunoprecipitation. A total of $30 \mu \mathrm{l}$ of Protein G Magnetic Beads (Millipore) were washed $3 x$ in immunoprecipitation buffer and then incubated in $1 \mathrm{ml}$ of immunoprecipitation buffer with $1 \mu \mathrm{g}$ of antibody for $4 \mathrm{~h}$ at $4^{\circ} \mathrm{C}$ under rotation. $10 \times 10^{6}$ cells were lysed in $500 \mu \mathrm{l}$ of immunoprecipitation buffer $(20 \mathrm{mM}$ Tris-HCl (pH7.5), $137 \mathrm{mM} \mathrm{NaCl}, 1 \%$ Triton X-100, $2 \mathrm{mM}$ EDTA (pH 8)) containing complete protease inhibitor cocktail and incubated for $30 \mathrm{~min}$ in ice. A total of $1400 \mu \mathrm{g}$ of cell extract were incubated overnight in $1 \mathrm{ml}$ of immunoprecipitation buffer with the antibody-coupled beads. The next day, beads were washed five times with immunoprecipitation buffer and eluted with $60 \mu \mathrm{l}$ of immunoprecipitation buffer containing $2 \%$ SDS. Then $20 \mu \mathrm{l}$ of laemmli buffer $4 \times$ were added, and samples were boiled for $10 \mathrm{~min}$ at $95^{\circ} \mathrm{C}$. Eluted proteins were split in two gels of SDS-polyacrylamide gel electrophoresis. Ten percent of the total protein subjected to immunoprecipitation was loaded as input and $30 \mu \mathrm{l}$ of the remaining supernatant after immunoprecipitation was also loaded to confirm immunodepletion. A total of $1 \mu \mathrm{g}$ of anti-HA and anti-ApoL2 described above were used for immunoprecipitation.

Statistics. Error bars in the figures represent the standard error of the mean (S.E.M.). Data were statistically analyzed to find significant differences using twotailed, paired Student's $t$-test. Significant differences are marked in the figures with ${ }^{*}(P \leq 0.05)$ or ${ }^{* *}(P \leq 0.0005)$.

\section{Conflict of Interest}

The authors declare no conflict of interest.

Acknowledgements. We wish to thank Dorothée Walter, Silvia RamírezPeinado, Dídac Domínguez and Clara León-Annicchiarico for help with experiments and Jean-Ehrland Ricci, Seamus Martin, Giulio Donati, Albert Tauler, Oscar M Tirado, Fabien Llambi, Pat Fitzgerald and Doug Green for plasmids, reagents and/ or advice. This work was supported by the Association for International Cancer Research (AICR), grant number 08-0621 and Fondo de Investigaciones Sanitarias of Spain, grant numbers PI10/00104 and Pl13/00139.

1. Droin NM, Green DR. Role of Bcl-2 family members in immunity and disease. Biochim Biophys Acta 2004; 1644: 179-188.

2. Frenzel A, Grespi F, Chmelewskij W, Villunger A. Bcl2 family proteins in carcinogenesis and the treatment of cancer. Apoptosis 2009; 14: 584-596.

3. Lomonosova $\mathrm{E}$, Chinnadurai $\mathrm{G}$. BH3-only proteins in apoptosis and beyond: an overview. Oncogene 2008; 27: S2-S19.

4. Youle RJ, Strasser A. The BCL-2 protein family: opposing activities that mediate cell death. Nat Rev Mol Cell Biol 2008; 9: 47-59.

5. Wan G, Zhaorigetu S, Liu Z, Kaini R, Jiang Z, Hu CA. Apolipoprotein L1, a novel Bcl-2 homology domain 3-only lipid-binding protein, induces autophagic cell death. J Biol Chem 2008; 283: 21540-21549.

6. Zhaorigetu S, Wan G, Kaini R, Jiang Z, Hu CA. ApoL1, a BH3-only lipid-binding protein induces autophagic cell death. Autophagy 2008; 4: 1079-1082.

7. Liu Z, Lu H, Jiang Z, Pastuszyn A, Hu CA. Apolipoprotein I6, a novel proapoptotic Bcl-2 homology 3-only protein, induces mitochondria-mediated apoptosis in cancer cells. $\mathrm{Mol}$ Cancer Res 2005; 3: 21-31.

8. Perez-Morga D, Vanhollebeke B, Paturiaux-Hanocq F, Nolan DP, Lins L, Homble F et al. Apolipoprotein $\mathrm{L}-\mathrm{I}$ promotes trypanosome lysis by forming pores in lysosomal membranes. Science 2005; 309: 469-472.
9. Vanhollebeke B, Pays E. The function of apolipoproteins L. Cell Mol Life Sci 2006; 63: 1937-1944.

10. Sigrist CJA, de Castro E, Cerutti L, Cuche BA, Hulo N, Bridge A et al. New and continuing developments at PROSITE. Nucleic Acids Res 2013; 41: D344-D347.

11. Finn RD, Bateman A, Clements J, Coggill P, Eberhardt RY, Eddy SR et al. Pfam: the protein families database. Nucleic Acids Res 2014; 42: D222-D230.

12. Coultas L, Pellegrini M, Visvader JE, Lindeman GJ, Chen L, Adams JM et al. Bfk: a novel weakly proapoptotic member of the $\mathrm{Bcl}-2$ protein family with a $\mathrm{BH} 3$ and a $\mathrm{BH} 2$ region. Cell Death Differ 2003; 10: 185-192.

13. Kaczmarek K, Studencka M, Meinhardt A, Wieczerzak K, Thoms S, Engel W et al. Overexpression of peroxisomal testis specific 1 protein induces germ cell apoptosis and leads to infertility in male mice. Mol Biol Cell 2011; 22: 1766-1779.

14. Liao W, Goh FY, Betts RJ, Kemeny DM, Tam J, Bay BH et al. A novel anti-apoptotic role for apolipoprotein L2 in IFN-gamma-induced cytotoxicity in human bronchial epithelial cells. J Cell Physiol 2011; 226: 397-406.

15. Ahn WS, Bae SM, Lee JM, Namkoong SE, Han S-J, Cho YL et al. Searching for pathogenic gene functions to cervical cancer. Gynecol Oncol 2004; 93: 41-48.

16. Page NM, Butlin DJ, Lomthaisong K, Lowry PJ. The human apolipoprotein $\mathrm{L}$ gene cluster: identification, classification, and sites of distribution. Genomics 2001; 74: 71-78.

17. Zhaorigetu S, Yang Z, Toma I, McCaffrey TA, Hu CA. Apolipoprotein L6, induced in atherosclerotic lesions, promotes apoptosis and blocks Beclin 1-dependent autophagy in atherosclerotic cells. J Biol Chem 2011; 286: 27389-27398.

18. Llambi F, Moldoveanu T, Tait SW, Bouchier-Hayes L, Temirov J, McCormick LL et al. A unified model of mammalian $\mathrm{BCL}-2$ protein family interactions at the mitochondria. Mol Cell 2011; 44: 517-531.

19. Aouacheria A, Brunet F, Gouy M. Phylogenomics of life-or-death switches in multicellular animals: Bcl-2, BH3-only, and BNip families of apoptotic regulators. Mol Biol Evol 2005; 22 2395-2416.

20. Duchateau PN, Pullinger CR, Orellana RE, Kunitake ST, Naya-Vigne J, O'Connor PM et al. Apolipoprotein L, a new human high density lipoprotein apolipoprotein expressed by the pancreas. Identification, cloning, characterization, and plasma distribution of apolipoprotein L. J Biol Chem 1997; 272: 25576-25582.

21. Monajemi H, Fontijn RD, Pannekoek $H$, Horrevoets AJ. The apolipoprotein L gene cluster has emerged recently in evolution and is expressed in human vascular tissue. Genomics 2002; 79: 539-546.

22. Kuwana T, Mackey MR, Perkins G, Ellisman MH, Latterich M, Schneiter R et al. Bid, Bax and lipids cooperate to form supramolecular openings in the outer mitochondria membrane. Cell 2002; 111: 331-342.

23. Wu X, Wang H, Bai L, Yu Y, Sun Z, Yan Y et al. Mitochondrial proteomic analysis of human host cells infected with H3N2 swine influenza virus. J Proteomics 2013; 91 136-150.

24. Tischner D, Villunger A. Bcl-G acquitted of murder! Cell Death Dis 2012; 3: e405

25. Giam M, Okamoto T, Mintern JD, Strasser A, Bouillet P. Bcl-2 family member Bcl-G is not a proapoptotic protein. Cell Death Dis 2012; 3: e404.

26. Hetz C, Glimcher L. The daily job of night killers: alternative roles of the BCL-2 family in organelle physiology. Trends Cell Biol 2008; 18: 38-44.

27. Laulier C, Lopez BS. The secret life of Bcl-2: apoptosis-independent inhibition of DNA repair by Bcl-2 family members. Mutat Res 2012; 751: 247-257.

28. Russell DJ, Katoh K, Standley D. MAFFT: iterative refinement and additional methods In: Multiple Sequence Alignment Methods. Humana Press: Suita, Japan, 2014 pp 131-146.

29. Capella-Gutiérrez S, Silla-Martínez JM, Gabaldón T. trimAl: a tool for automated alignment trimming in large-scale phylogenetic analyses. Bioinformatics 2009; 25: 1972-1973.

30. Eddy SR. Accelerated profile HMM searches. PLoS Comput Biol 2011; 7: e1002195.

31. Flicek P, Amode MR, Barrell D, Beal K, Billis K, Brent S et al. Ensembl 2014. Nucleic Acids Res 2014; 42: D749-D755.

32. Slater GS, Birney E. Automated generation of heuristics for biological sequence comparison. BMC Bioinformatics 2005; 6: 31 .

(i) (2) $\Theta$ Cell Death and Disease is an open-access journal published by Nature Publishing Group. This work is licensed under a Creative Commons Attribution-NonCommercialNoDerivs 3.0 Unported License. The images or other third party material in this article are included in the article's Creative Commons license, unless indicated otherwise in the credit line; if the material is not included under the Creative Commons license, users will need to obtain permission from the license holder to reproduce the material. To view a copy of this license, visit http://creativecommons.org/licenses/ by-nc-nd/3.0/ 